\title{
Modelling and Simulation of Kinetic Energy and it Impact on the Conservation Of Energy in an Automotive Hydraulic Shock Absorber during Damping Process
}

\author{
P. B. Sob \\ Department of Mechanical Engineering, Faculty of Engineering and Technology, \\ Vaal University of Technology, Vanderbijlpark 1900, Private Bag X021, South Africa.
}

\begin{abstract}
In the current study the kinetic energy stored in a hydraulic shock absorber during damping is modelled theoretical. The theoretical model takes into account the relevant parameters that affect the kinetic energy of the damper during damping process. The fluid viscosity, density and temperature of the hydraulic fluid during damping is taken into consideration during modelling and simulation process. The kinetic energy harvested during damping process is revealed in the current study. The theoretical study was performed by simulating a twin tube damper system using the tool of SOLIDWORKS 2017-software. The physical behaviour of kinematics energy, density, viscosity and temperatures of fluid in the damping process was modelled theoretically. The following facts were theoretically revealed during the modelling process. It was shown that an increase in the system's velocity resulted to an increase in the system's Kinetic Energy. The results also revealed that even though the vehicle's mass is constant, the vehicle's Kinetic Energy is at its highest when the vehicle's dampers dampens vibratory oscillations at high velocities while transferring the energy of the spring motion to the damper whist ensuring ride comfort. It was also revealed from the pressure simulation results that the pressure difference between iterations $0-5(\mathrm{t})$ showed a large drop from iterations 5-40(t) and the pressure experienced fluctuations of maximum and minimal peaks until it stabilized during damping process. It was also revealed from the geometry of the damping system and fluid behaviour during damping operations that the drop in pressure is highly controlled by the resistance of fluid flow inside the damper. This resistance is caused by the change in geometry between the working cylinder and reserve cylinder as the fluid experienced change in velocities thus experienced turbulence as it entered and exit the change in geometry of the damper from the working cylinder to the reserve cylinder.
\end{abstract}

Keywords: efficiency, temperature, flow rate, evaporator and stability.

\section{INTRODUCTION}

The hydraulic damper assist in protecting the components of the vehicle during oscillation process and also ensures a better ride quality, it absorbs and damps the shock impulses by converting kinetic energy, caused by the spring of the suspension system, into thermal energy. During damping process the hydraulic fluid inside the hydraulic damper experiences transformations. These transformations are caused by variations experienced by the fluid due to pressure changes in the system that results from the piston displacement, which in turn generates heat energy that is dissipated. The dissipated heat results in a loss of energy that could be otherwise conserved to power alternative mechanical systems in the vehicle. Recent studies revealed that there is more demand for energy optimising in the automotive industries, specifically clean energy (Environmental Science, 2018). Therefore the study of the hydraulic energy harvesting in a hydraulic damper is vital since it can power other mechanical and electrical component in an automotive system.

Previous studies in automotive hydraulic shock absorber developed damping models based on damper velocity, force and displacement parameters during damping process (Naire S, 2013), without considering other major energy parameter of the damper system during operation such as kinetic energy, potential energy, density of fluid, pressure during damping and change in temperature which affect the fluid viscosity. Therefore the derived models are inefficient in capturing the damping energy produced in a car damping system. For this reason the developed models have limitations to serve as a guide for selecting suitable Energy Regenerative Systems which can recover the wasted energy hereby making use of the otherwise wasted Energy, since Energy sources are exhaustible. In the current study the ignored parameters are considered in modelling damping behaviour of an automotive damper. The obtained results are analysed to show results that are ignored by other derived models of energy generation that considered limited parameters.

\section{MODELLING AND SIMULATING DAMPING PROCESS DURING OSCILLATION CYCLES}

In modelling the conservation of energy during damping process the following parameters are used in modelling and simulation process, Fluid Pressure $\left(\boldsymbol{P}_{1}, \boldsymbol{P}_{\mathbf{2}}\right)$, Fluid Density $\left(\boldsymbol{\rho}_{1}, \boldsymbol{\rho}_{2}\right)$ and Fluid Velocity $\left(\boldsymbol{V}_{\mathbf{1}}, \boldsymbol{V}_{\mathbf{2}}\right)$. The test were conducted through parametric experimentation and the theoretical simulation using SOLIDWORKS 2017. The simulation was 
conducted for the validation of the proposed models.

\section{PARAMETRIC ANALYSIS OF KINETIC ENERGY DURING DAMPING PROCESS}

During the damping behaviour of the damper, Kinetic Energy is defined as the Energy the damper has due to its motion. It is directly proportional to the system's velocity and mass. Now if a system of constant mass i.e. a midsize vehicle was taken and allowed to undergo a change in velocity at any given time, the Kinetic Energy graph of the system can be plotted which can describe the system's total Kinetic Energy during motion. The table below was used to plot the Kinetic Energy of the midsize vehicle. The mass of the vehicle during normal operation when simulation was performed was $1590 \mathrm{~kg}$.

Table 1: Kinetic Energy of the system during damping.

\begin{tabular}{|c|c|}
\hline Vehicle Velocity $(\mathrm{m} / \mathrm{s})$ & Total Kinetic Energy $(\mathrm{J})$ \\
\hline 40 & 1272 \\
\hline 42 & 1402.38 \\
\hline 50 & 1987.50 \\
\hline 55 & 2404.88 \\
\hline 60 & 2862 \\
\hline
\end{tabular}

From table 1 it was observed that during damper operation an increase in velocity of the vehicle lead to an increase in the Kinetic Energy of the system. This observation showed that the system's potential to generate Kinetic Energy is increased at higher velocities. The geometry of the graph was than plotted.

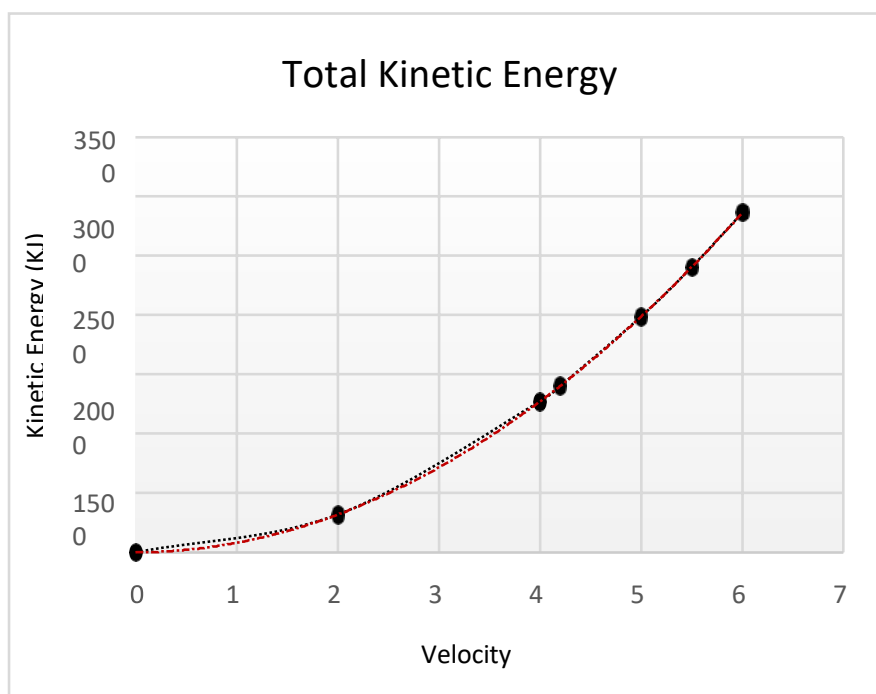

Figure 1: Kinetic Energy graph of the system during operation.

From the above graph, the geometry of the vehicle's Kinetic Energy reflected a parabolic trend. The graph also showed that an increase in the system's velocity resulted in an increase in the system's total Kinetic Energy, this result was of expectation as the Kinetic Energy is dependent and directly proportional to the system's mass and velocity. The trend further reflected that even though the vehicle's mass is constant, the vehicle's Kinetic Energy is at its highest when the vehicle's dampers dampens vibratory oscillations at high velocities while transferring the energy of the spring motion to the damper whist ensuring ride comfort, as illustrated by the excitation and response portion encircled, in figure 2. In this figure the function of the damper was shown as it served to damp out vibratory oscillations. In figure 2 the magnitude of the vibratory excitation was reduced towards the response side of the vehicle. This concept was related to the velocity of the fluid of the damper system as the fluid enters and exits the reserve cylinder during damping operation. The initial velocity of the fluid was directly proportional to the velocity of the piston as the damping effectiveness related to the speed at which the system travels. The faster the piston travelled thus fluid travelled, the quicker the oscillations were dampened. The Kinetic Energy of the Conservation of Energy Model should thus reflect a similar trend. It was hereby also important to analyse the shape of the Potential Energy of the system to understand the expected relation of the developed Model's Potential Energytrend.

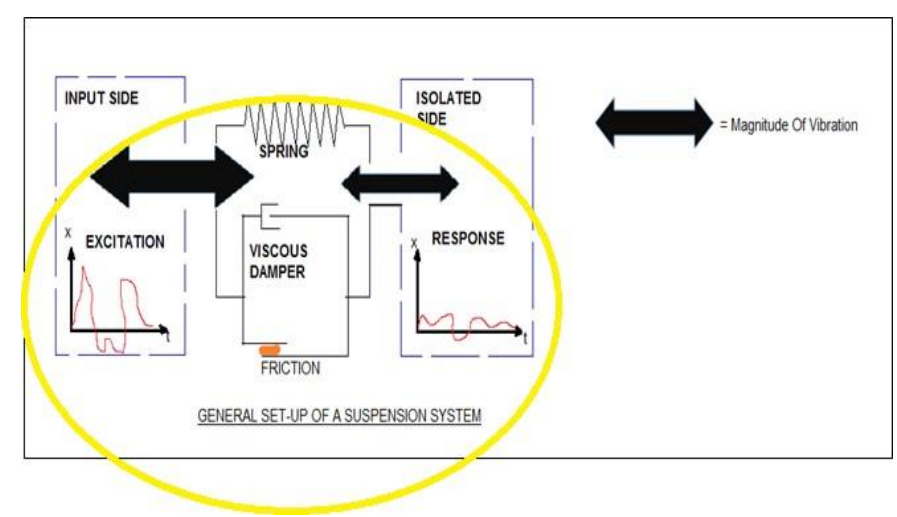

Figure 2: General Setup of a Suspension System.

\section{ANALYSIS OF PROPOSED MODELS}

From the modified Mechanical Energy Model, it was noted that the Kinetic Energy of the model depends on the fluid's density, change in volume, $\Delta \boldsymbol{V}$ and velocity $\boldsymbol{V}_{2}$. To obtain these parameters the concept of compressible flow was of importance and the thermodynamic process, isothermal process, experienced by the fluid operation became useful. From the chart in figure 3 , the relationship between density, and pressure of the system during damper operation is shown. The concept was focussed on the behaviour of the fluid under the parameters analysed i.e. density $(\boldsymbol{\rho})$ and pressure $(\mathrm{P})$. The chart further reflected that with a change in pressure came a change in density which was of adherence to the concept of compressible flow. It was noted that the change of density with pressure parameter has not been explored in previous studies, henceforth it became the core study in this project. As the assumption was made that the damper system is described by an Isothermal thermodynamic process because no external heat is added to the system, the chart reflected that the density of the fluid experienced a slight change at high pressures. This further placed emphasis that during damper operation the density of the fluid cannot be taken as being constant, as was the case in previous studies. 
International Journal of Engineering Research and Technology. ISSN 0974-3154, Volume 13, Number 7 (2020), pp. 1575-1583

(C) International Research Publication House. https://dx.doi.org/10.37624/IJERT/13.7.2020.1575-1583

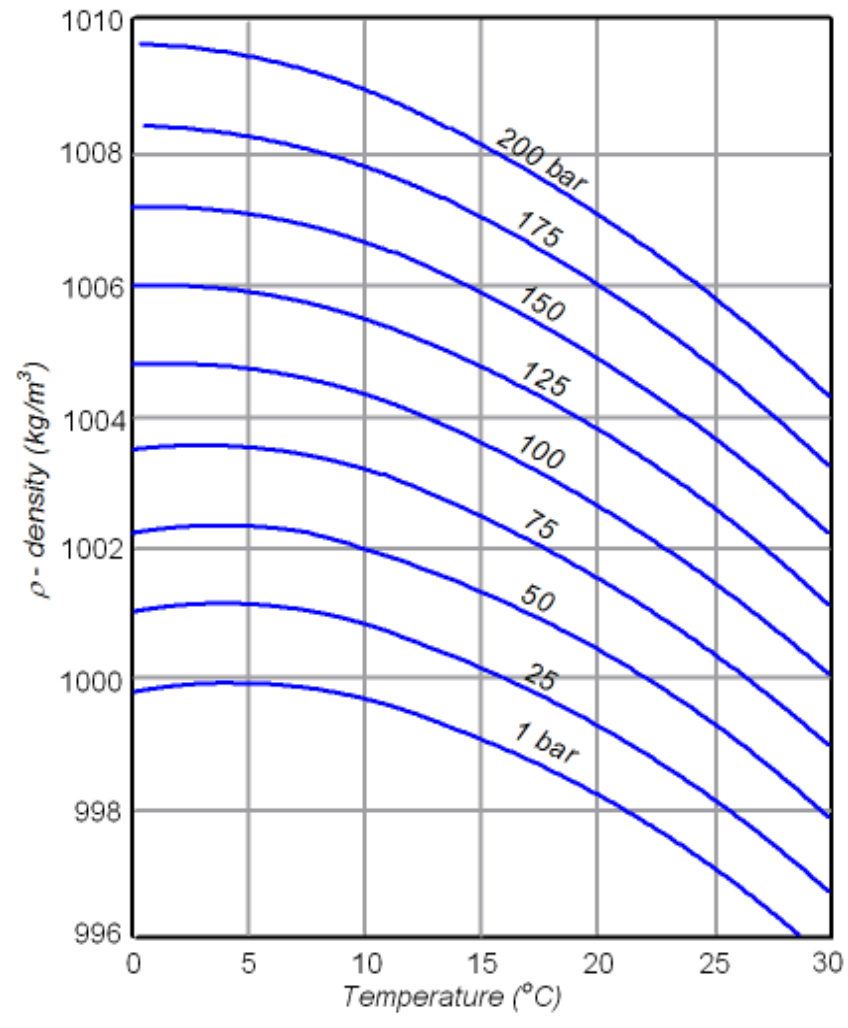

engineeringtoolbox.com

Figure 3: Pressure Density Chart of the damper system during operation.

The density, $\boldsymbol{\rho}_{2}$, and velocity, $\boldsymbol{V}_{\mathbf{2}}$ of the Kinetic Energy Model is the density and velocity of the fluid at the entrance of the reserve cylinder. It is at this section where a change in pressure and thus change in density occurs. This point is shown by (c) in the damper diagram and by (d) in the simplified version of the damper system as shown in figure 4 .
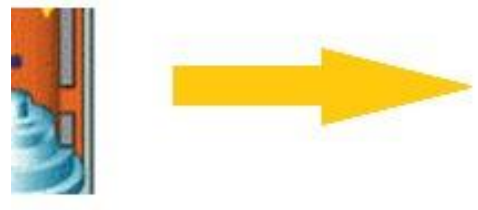

C

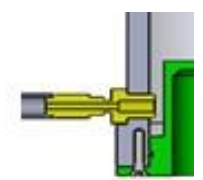

d
Figure 4: Entrance of the fluid to the Reserve cylinder during damper operation.

zAfter the discussion of the fundamental concepts and parameters, the analysis of the results was done. The simulation of the Twin Tube Damper system was conducted for validation of the theoretical concept discussed. However the geometry of the damper system also served of importance and thus was analysed.

\section{MODEL GEOMETRY ANALYSIS}

For the analysis of modelling, the characteristic of the fluid flow between two points of different cross sectional area was considered since the fluid flow during damper operation experiences the same pattern. The following theoretical study related to the fluid flow in figure 5.

(a)

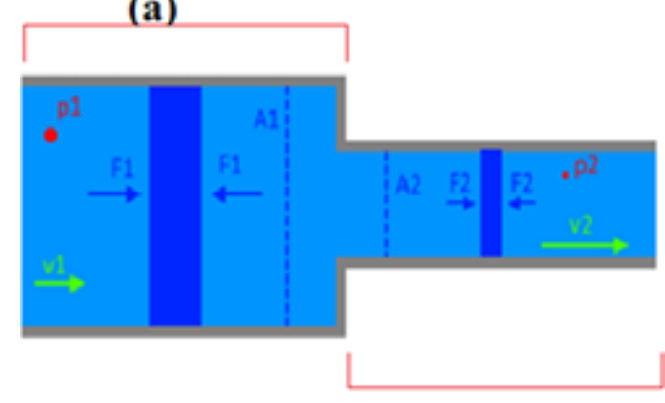

(b)

Figure 5: Flow of fluid between different areas of the damper system.

The following assumption and parameters are used in modelling and simulation. A change in cross section, $A_{2}<A_{1}$. From the law of conservation of mass flow from section A1 and A2 their change in velocity of flow in $A_{2}<A_{1}$ is given as $v_{1}<$ $v_{2}$ and from Bernoulli their change in pressure is given as $p_{2}<$ $p_{1}$ and from continuity their flow rate is given as, $Q_{1}=Q_{2}=A v$. where

$A=$ Cross sectional area of geometry change (m2), $v=$ Velocity of fluid during operation $(\mathrm{m} / \mathrm{s}), P_{1}=$ Pressure in the pressure cylinder of the damper $(\mathrm{Pa}), P_{2}=$ Pressure in the reserve cylinder of the damper $(\mathrm{Pa}), Q_{1}=Q_{2}$ Flow rate of fluid (m3/s). These changes described the changes of the considered parameters which served as guidance during the Modelling and Simulation process. Normally during vehicle operation, as the vehicle tire drops in the pothole the vehicle is no longer level and the vehicle weight begins to swift more severely over the tire. Therefore the force of impact which the vehicle hits the pothole is directly proportional to the velocity at which the vehicle travels. This can be related to one of the Newtonian laws of motion which is mass and acceleration is directly proportional to the applied force given as $\mathrm{F}=$ maand $\mathrm{a}=\mathrm{F} / \mathrm{m}$, where $\mathrm{F}$ is the force of impact, and $\mathrm{m}$ the vehicle mass and "a" the vehicle acceleration. And the piston velocity $V_{\text {piston }}=V_{o}+a t$, where $V_{\text {piston }}$ is the velocity of piston during impact, $V_{o}$ the initial velocity of piston before impact and $t$ is the set time frame. From the relation of the two above mentioned equations, the following results were obtained. Under these results the time frame of impact was set to be 1 second since the aim was to study how the force of impact influenced the velocity at which the piston travelled. The mass of the vehicle, during damper operation, was that of a midsize vehicle i.e. $1590 \mathrm{~kg}$.

Table 2: Relationship of the piston force and velocity during damper operation.

\begin{tabular}{|c|c|c|c|}
\hline $\begin{array}{c}\text { Vehicle velocity } \\
(\mathrm{m} / \mathrm{s})\end{array}$ & Force ofimpact (N) & Time (s) & V piston $(\mathrm{m} / \mathrm{s})$ \\
\hline 70 & 500 & 1 & 0.314 \\
\hline 90 & 600 & 1 & 0.377 \\
\hline 100 & 650 & 1 & 0.409 \\
\hline 110 & 700 & 1 & 0.440 \\
\hline
\end{tabular}

Table 2 showed that the magnitude of the force at which the 
vehicle's wheels experiences impact is directly proportional to the velocity at which the vehicle travels. At high velocities the force of impact was increased. The relation between the impact force and the damper piston velocity resulted in the study that the damper piston velocity increases as the impact force increased. As the velocity increased, the impact force also increased at higher vehicle velocities. The piston equally travels faster completing more strokes thus dampening out more oscillations than when the vehicle travels at lower speeds. The increased stroke completion resulted in the damper system doing more work at a faster rate hereby experiencing higher energy dissipation which can be conserved over time. From this result it was noted that depending on the surface of the road, at higher vehicle velocity the damper system dissipates more energy while performing its function of dampening oscillations. It was with this information together with the assumptions to follow that the graphs of the Kinetic and Potential Energy of the modified Mechanical Energy Model were plotted. The geometry of these plots was then be compared for analysis based on few assumption. Assumption 1: During impact the fluid in the working cylinder travels with the same velocity at which the piston travels. Assumption 2: The fluid properties used are those at constant temperature, $15^{\circ} \mathrm{C}$, as per chart in figure 3 . The analysis of the system is conducted while considering the compression stroke of the damper system during operation. The following application are analyse during modelling and simulation. 1. The Kinetic Energy Model graph. According to the diagram in appendix A, the area of the piston on which the force acts was obtained by using the equation : $A_{1}=\frac{\pi d^{2}}{4}$ where : $A_{1}$ is the piston area and $\mathrm{d}$ is the working's piston's diameter. From the data obtained in table 2, the pressure: $P_{1}=\frac{F}{A_{1}}$ where $\mathrm{F}$ is the impact force and $A_{1}$ is the piston area. These expressions revealed that the impact at which the vehicle hits a pothole results in a velocity, $V_{1}$ and pressure, $P_{1}$, which are the parameters during the initial movement of the piston and thus the fluid's motion shown by (a) in figure 5. Using the chart in figure 3 and applying interpolation, the density at this pressure, ${ }_{1}$ at constant temperature was obtained which was further used to get the pressure, $P_{2}$ at part (b) of figure 5. By interpolation and with reference to the chart, the following results were tabulated in table 3 . The first points of density and pressure read was 1 bar and $999 \mathrm{~kg} / \mathrm{m}^{\wedge} 3$ and the second points hereof was 25 bar and $1000.1 \mathrm{~kg} / \mathrm{m}^{\wedge} 3$.

Table 3: Parameters of fluid at initial motion of damper.

\begin{tabular}{|c|c|c|c|c|}
\hline $\begin{array}{c}\text { Impact } \\
\text { forc } \\
\text { e (N) }\end{array}$ & $\begin{array}{c}\text { Area } \\
\left(\boldsymbol{*}^{\mathbf{1 0}} \mathbf{6}^{-\mathbf{m}} \boldsymbol{m}^{\mathbf{2}}\right)\end{array}$ & $\begin{array}{c}\text { Velocity, } \\
\boldsymbol{V}_{\mathbf{1}}\left(\begin{array}{c}\text { m/s) } \\
\text { m }\end{array}\right.\end{array}$ & $\begin{array}{c}\text { Pressure, } \\
\boldsymbol{P}_{\mathbf{1}} \\
(\text { bar })\end{array}$ & $\begin{array}{c}\left.\text { Density, } \boldsymbol{\rho}_{\mathbf{1}}\right) \\
\mathrm{kg} / \mathrm{m}^{\wedge} 3 .\end{array}$ \\
\hline 500 & 962.113 & 0.314 & 5.196 & 999.19 \\
\hline 600 & 962.113 & 0.377 & 6.236 & 999.24 \\
\hline 650 & 962.113 & 0.409 & 6.756 & 999.26 \\
\hline 700 & 962.113 & 0.440 & 7.276 & 999.29 \\
\hline
\end{tabular}

From table 3 , the area, $A_{1}$ pressure, $P_{1}$ and density, $\rho_{1}$ were of interest since the relation between the impact force, $F$, and velocity, ${ }_{1}$ has been discussed. The trend in the relation between the impact force, area and pressure reflected that even though a damper system can have a constant geometry with constant cross sectional area, the force at which the vehicle tire experiences impact has an effect on the pressure which built-up in the fluid. The results obtained revealed that the change in density is directly proportional to the change in pressure.

Henceforth it was concluded that the compressibility of the hydraulic fluid in the working cylinder, during damping, is dependent on the impact force since this force affects the parameters of interest of compressible flow i.e. fluid pressure and fluid density. This observation revealed how the external force affected the internal parameters of the fluid during damping operation and thus its compressibility. The increase in the fluid's compressibility rate further reflected that at a high force the damper converts a high amount of Kinetic Energy from spring oscillations to thermal heat, dissipating more Energy.

The parameter's i.e. density,2, velocity, $V_{2}$ and pressure, $P_{2}$ relation at point (b) of figure 5 was obtained by applying the concept of fluid continuity between areas (a) and (b) of figure 5 . From this concept the velocity, $V_{2}$ was obtained and by using knowledge of the fluid's compressibility the density, $\rho_{2}$ was obtained. This concept is expressed by the equation: $Q_{1}=Q_{2}$, $A_{1} v_{1}=A_{2} v_{2}$ and $A_{1} v_{1} \rho_{1}=A_{2} v_{2} \rho_{2}$ where $Q_{1}$ and $Q_{2}$ is the fluid flow rate under normal condition, $A_{1}, A_{2}$ is the Areas at point (a) and (b) of figure 5 and $v_{1}, v_{2}$ is the velocity of fluid during operation. Pressure, $P_{2}$ was obtained by the application of the isothermal process theory under compressible flow, which was obtained from: $P_{2}=\frac{P_{1} \rho_{2}}{\rho_{1}}$ where $P_{1} . \rho_{1}$ is the pressure density of fluid at area (a) of figure 4.6 during damping, and $P_{2}, \rho_{2}$ is the pressure and density of fluid at area (b) of figure 5. By application of these equations at point (b) of figure 5 table 4 was obtained.

Table 4: Parameters of fluid at entrance of reserve cylinder.

\begin{tabular}{|c|c|c|c|c|c|c|c|}
\hline $\begin{array}{l}\text { Pressure, } \\
\boldsymbol{P}_{\mathbf{1}} \text { (bar) }\end{array}$ & $\begin{array}{c}\left.\text { Density, } \boldsymbol{\rho}_{1}\right) \\
\mathrm{kg} / \mathrm{m}^{\wedge} 3 .\end{array}$ & $\begin{array}{c}\text { Areal } \\
\left(\mathbf{1 0}^{-6} \mathbf{m}^{2}\right)\end{array}$ & $\begin{array}{c}\text { Velocity } \\
\boldsymbol{V}_{1} \\
(\mathrm{~m} / \mathrm{s})\end{array}$ & $\begin{array}{c}\text { Area2 } \\
\left(\mathbf{* 1 0}^{-6} \mathbf{m}^{\mathbf{2}}\right)\end{array}$ & $\begin{array}{c}\text { Velocity, } \\
\boldsymbol{V}_{\mathbf{2}} \\
(\mathrm{m} / \mathrm{s})\end{array}$ & $\begin{array}{c}\text { Density, } \\
\left(\boldsymbol{\rho}_{2}\right) \\
\mathrm{kg} / \mathrm{m}^{\wedge} 3 .\end{array}$ & $\begin{array}{l}\text { Pressure, } \\
\boldsymbol{P}_{2} \text { (bar) }\end{array}$ \\
\hline 5.196 & 999.19 & 926.113 & 0.314 & 314.159 & 0.962 & 998.80 & 5.194 \\
\hline 6.236 & 999.24 & 926.113 & 0.377 & 314.159 & 1.155 & 998.86 & 6.234 \\
\hline 6.756 & 999.26 & 926.113 & 0.409 & 314.159 & 1.253 & 998.91 & 6.754 \\
\hline 7.276 & 999.29 & 926.113 & 0.440 & 314.159 & 1.348 & 998.92 & 7.273 \\
\hline
\end{tabular}

The obtained results revealed that during damper operation, the hydraulic fluid in the damper cylinders undergoes a change in velocity whereby the velocity at the smaller cross sectional area is higher than the velocity at the larger cross sectional area, which is of expectation according to the principle of conservation of mass and fluid continuity. As the velocity increased, the pressure, $P_{2}$ at this point of increased velocity experienced a slight decrease compared to pressure (1). It was thus noted that the pressure at the reserve cylinder is inversely proportional to the fluid velocity. Even when there is a slight decrease in the pressure difference, both pressures experienced an increase of which the densities $\left(\rho_{1}, \rho_{2}\right)$ at these points similarly experienced an increase. The parametric experimental results related the operation of the Twin Tube damper system to the concept of the fluid's compressibility and thus the system's Energy conservation potential according to the principle of compressible flow. From the modified Mechanical Energy model, the only parameter outstanding to complete the 
model is the change in specific volume, and under compressible flow the specific volume is the reciprocal of density. This is expressed as: $V=\frac{1}{\rho}$ and the change hereof as $\Delta V=$ $\left(\frac{1}{\rho_{1}}-\frac{1}{\rho_{2}}\right)$ where $\Delta V$ is the change in volume. By using the above equation, the change in volume, $\Delta V$ during damper operation and thus the Kinetic Energy of the system was obtained and tabulated in table 5 .

Table 5: Kinetic Energy of Modified Model.

\begin{tabular}{|c|c|c|c|}
\hline $\begin{array}{c}\text { Density, } \\
\mathrm{kg} / \mathrm{m}^{\wedge} 3 .\end{array}$ & $\begin{array}{c}\text { Density, } \\
\mathrm{kg} / \mathrm{m}^{\wedge} 3 .\end{array}$ & $\begin{array}{c}\Delta \mathrm{V} \\
\left(\mathrm{kg} / \mathrm{m}^{\wedge} 3\right)\end{array}$ & $\begin{array}{c}\text { Total } \\
\text { Kinetic } \\
\text { Energy }(\mathrm{J})\end{array}$ \\
\hline 999.19 & 998.80 & 0.391 & 180.71 \\
\hline 999.24 & 998.86 & 0.381 & 253.084 \\
\hline 999.26 & 998.91 & 0.351 & 275.236 \\
\hline 999.29 & 998.92 & 0.371 & 336.708 \\
\hline
\end{tabular}

It was observed that the Kinetic Energy increased as the change in volume decreased. This showed that as a drop in volume occurs due to a drop in the reciprocal of the fluid's density the fluid during operation travelled from the working cylinder to the reserve cylinder at an increased velocity thus experiencing turbulent flow whereby increased heat is dissipated. This observation was taken to be due to the internal energy of the molecules as they collide at high velocity rates, causing high Kinetic Energies which was dissipated as heat. At a high rate of molecular collisions, higher forces are generated at high velocities. Since the cross sectional area at the entrance to the reserve cylinder was smaller than that at the pressure cylinder, increased fluid turbulence resulted. This increased turbulence generates high internal fluid temperatures which is dissipated. The results hereof was plotted in figure 6 .

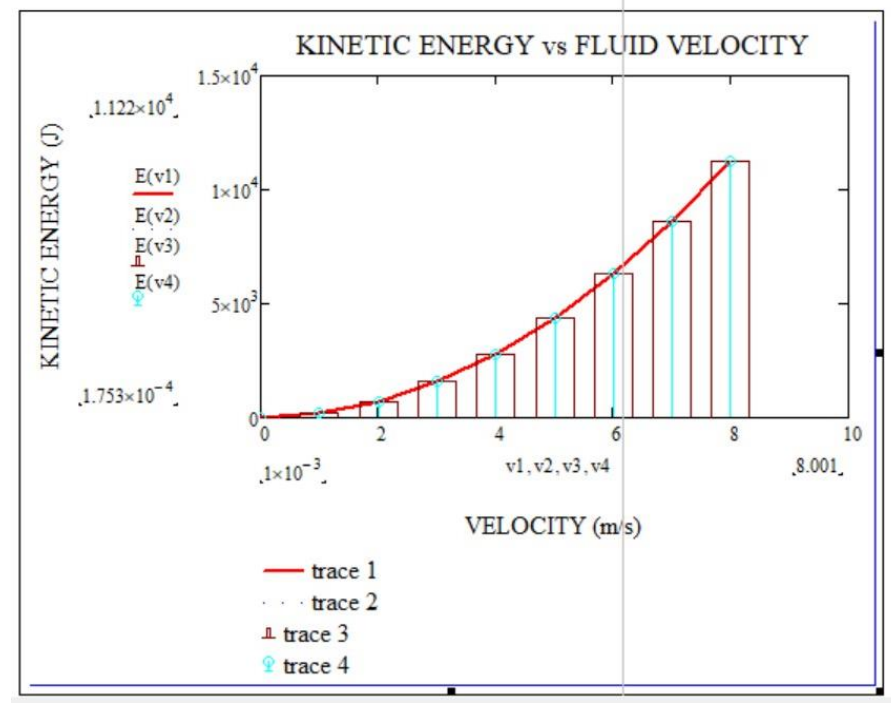

Figure 6: Kinetic Energy graph of Modified Model.

The Kinetic Energy graph was plotted and the geometry of the study is shown in figure 6 . From the graph $\mathrm{E}\left(v_{1}\right), \mathrm{E}\left(v_{2}\right), \mathrm{E}\left(v_{3}\right)$, $\mathrm{E}\left(v_{4}\right)$ represents the Kinetic Energy, shown by the traces, obtained as the fluid's density changes with the respective change in volume caused by change in pressure. A parabolic trend was obtained from the plot of the relation of the Kinetic Energy with respect to change in velocity. This result showed that the principle of compressible flow with focus on the change in pressure and thus change in density of the hydraulic fluid during damping operation can be used to obtain the Kinetic Energy of the damper system which is otherwise wasted through heat dissipation. At any instance during operation the pressure, $P_{2}$ can be used to obtain the fluid's density, $\rho_{2}$ and thus the velocity, $v_{2}$ which is used to obtain the Kinetic Energy of the system during operation. As the Kinetic Energy is dependent on the density, 2 change in volume, $\Delta \mathrm{V}$ and velocity, $v_{2}$, an increase or decrease of these parameters have an impact on the total Kinetic Energy conserved.

For optimal results of the Kinetic Energy, the system's parameters should be analysed when these parameters experience an increase which was obtained during the compression stroke of the damper system. At a fluid density, $\rho_{2}$ of $998.80 \mathrm{~kg} \cdot \mathrm{m}^{\wedge} 3$ and a velocity, $v_{2}$ of $0.962 \mathrm{~m} / \mathrm{s}$ the total Kinetic Energy of the damper was $180.71 \mathrm{~J}$ and at a density, $\rho_{2}$ of $998.92 \mathrm{~kg} / \mathrm{m}^{\wedge} 3$ and velocity of $1.340 \mathrm{~m} / \mathrm{s}$ the Kinetic Energy was $336.7 \mathrm{~J}$. This relationship revealed that at higher velocities and increased densities, an increased amount of Kinetic Energy was generated which can be used to power alternative mechanical functions of the vehicle rather than being lost. Even though the above graph represents the Kinetic Energy of the system at different densities, the trend is nearly similar- This is because the fluid experiences a slight increase in the pressure and thus density henceforth the traces of these parameters are closely plotted. From this result the modified Kinetic Energy Model was used to obtain and thus conserve the system's Kinetic Energy.

\section{RESULTS OF SIMULATION DERIVED MODELS}

The Simulation of the Twin Tube damper system was conducted in SOLIDWORKS 2017- Solid works is a software with analytical tools and design automations which helps to simulate physical behaviour of kinematics, dynamics, stress, deflection, vibration, and temperatures of fluid flow for engineering designs. It was in this program that the behaviour of the fluid under the set parameters was analysed. Since the fluid travels at the velocity initially transferred from the piston, the initial conditions of the fluid at entry point was that experienced by the piston during impact. These were velocity, $v_{1}=3.14 \mathrm{~m} / \mathrm{s}$ and pressure, $P_{1}=514600 \mathrm{pa}$. The following simulation analysis was conducted: 1 . The study of the fluid's change in pressure. 2 . The study of the fluid's density as the pressure changes. 3 . The study of velocity as the damper's geometry change and 4 . The study of the system's turbulent Energy.

During the simulation, the schematics of the system's performance had cut plot contours. The contours was used to observe how the response variables of the system during damping operation related to the predicted parameter variables The values for the response variables were represented by the shaded regions, the trend from the colour blue to red represented the increase of the response variables during system operation. These cut plot contours were found to be useful for 
investigating the desirable response variables. This simulation was of benefit for the validation of the selected models. The following simulation procedure was used

(a) The simplified version of the damper was drawn to set dimensions (b) The system's boundary conditions was set, which were the initial parameters of study (c) The goals to be achieved were selected to study the correlation of the theoretical models (d) During operation the system was calibrated to run at 0.1 seconds $(t)$. This was done to obtain accuracy of the results during operation, since the change in parameters happens simultaneously. The first results to be analysed was the fluid flow and pressure analysis.

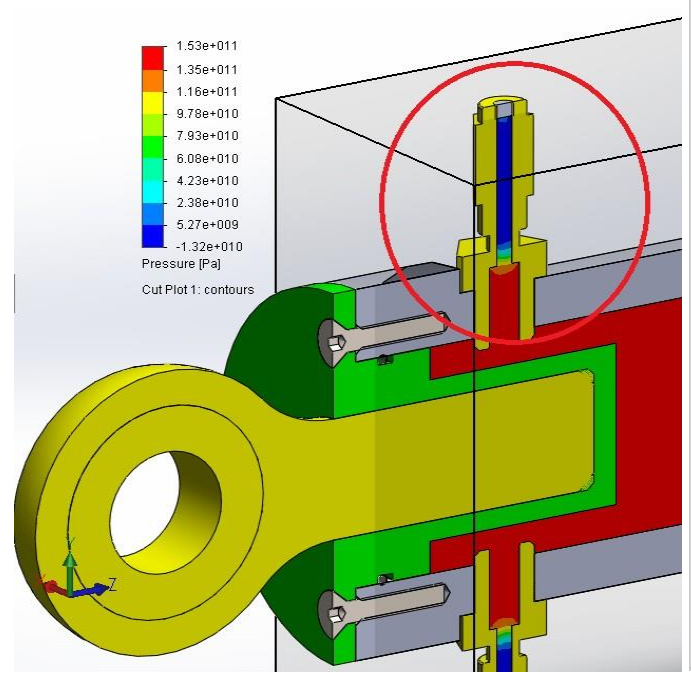

(a)

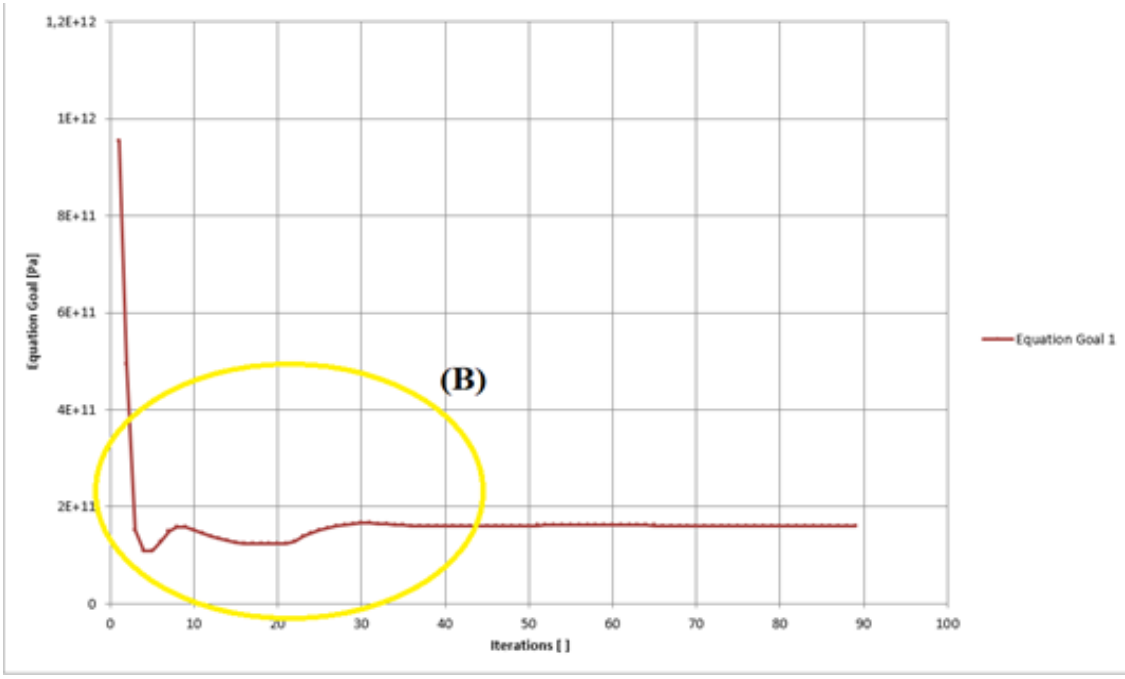

(b)

Figure 7 (a) Pressure simulation schematic with contour (b) Pressure Simulation Results.

From the pressure simulation results as shown in Fig.7 (a-b), it was observed that the pressure difference between iterations 0 $5(\mathrm{t})$ showed a large drop and from iterations 5-40(t) the pressure experienced fluctuations of maximum and minimal peaks until it stabilized, asshown by the encircled area marked B as shown in Fig.7 (b). By studying the geometry of the damper system and fluid behaviour during damping operations, the drop in the pressure can be caused by the resistance of fluid flow inside the damper system as shown in Fig. 7 (a). This resistance is caused by the change in geometry between the working cylinder and reserve cylinder as the fluid experienced change in velocities thus experienced turbulence as it entered and exit the change in geometry of the damper from the working cylinder to the reserve cylinder as shown in Figure 7 (a). This resulted in a pressure drop since pressure drop during turbulence is proportional to the square of the velocity $\left(\mathrm{P} \alpha V^{2}\right)$. The cylinder's wall roughness $(\varepsilon)$ also contributed to the pressure drop since the roughness effects the drag of the fluid where the drag between the fluids layers shears them apart, due to the viscous force $F_{v}$ and inertial force $F_{i}$, hereby resulting in each layer travelling at different speeds as shown in Fig. 7 (a). As the fluid layers travelled at different speeds it experienced turbulent flow which produced eddies that moved randomly inside the damper as the fluid entered and exit the reserve cylinder. During this occurrence the fluid experienced a transition from laminar to turbulent flow. All this resistance during the initial movement of the piston was high and had to be overcome by the fluid for the damper to perform its function effectively- Thus during the first iterations the drop was large and thereafter became minimal as the fluid overcome the resistance whilst operating. From iterations 5-40(t) the drop fluctuated and experienced a smaller drop as the fluid in motion overcome the initial high resistance and thus stabilized at small impact forces as shown in Fig. 7 (b). The next results to analyse was fluid velocity analysis during damping process in Figure 8. 


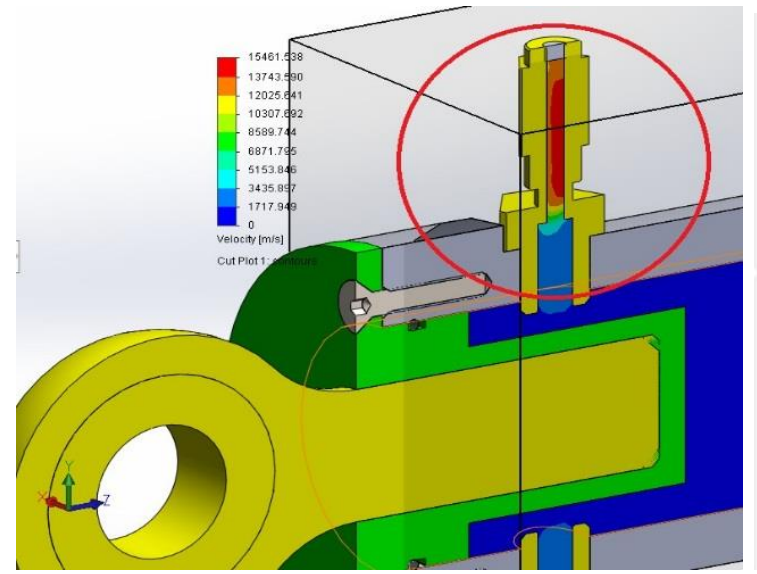

(a)

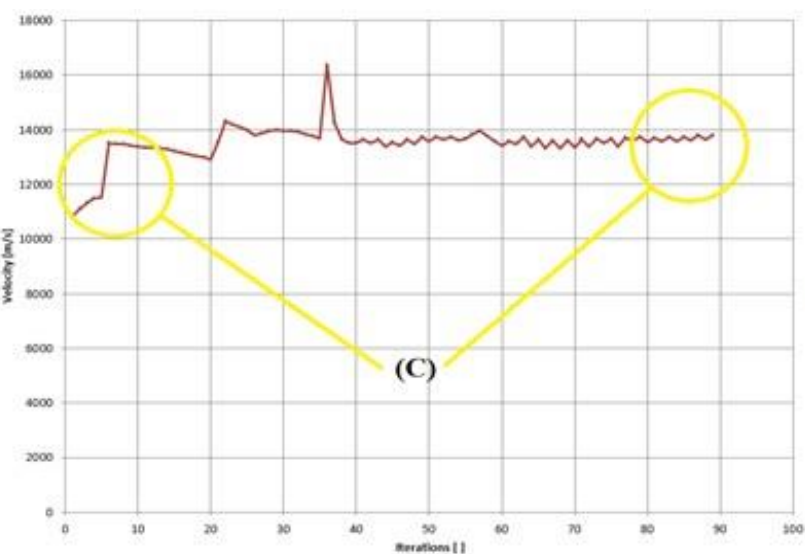

(b)

Figure 8 (a) Velocity simulation schematic with contours (b) Velocity and iteration

The geometry of the velocity simulation result showed an increase and decrease to a very high peak at approximately 35$40(\mathrm{t})$, this showed that during this iteration the damping was rough as shown in Figure 8 (b). This region is the same region of analysis as the pressure drop, meaning, as discussed, that the fluid during operation experiences high turbulence and an increased rate of velocity as the fluid travelled through the change of geometry as it enters and exits the reserve cylinder during operation as shown in Figure 8 (a). Since the change in geometry of the reserve cylinder as compared to the working cylinder allows a smaller area,

$A_{2}$ thus increased velocity, $v_{2}$ i.e. $\left(v_{2} / \alpha A_{2}\right)$. Both these factors has an impact on the fluctuating pattern of the increasing

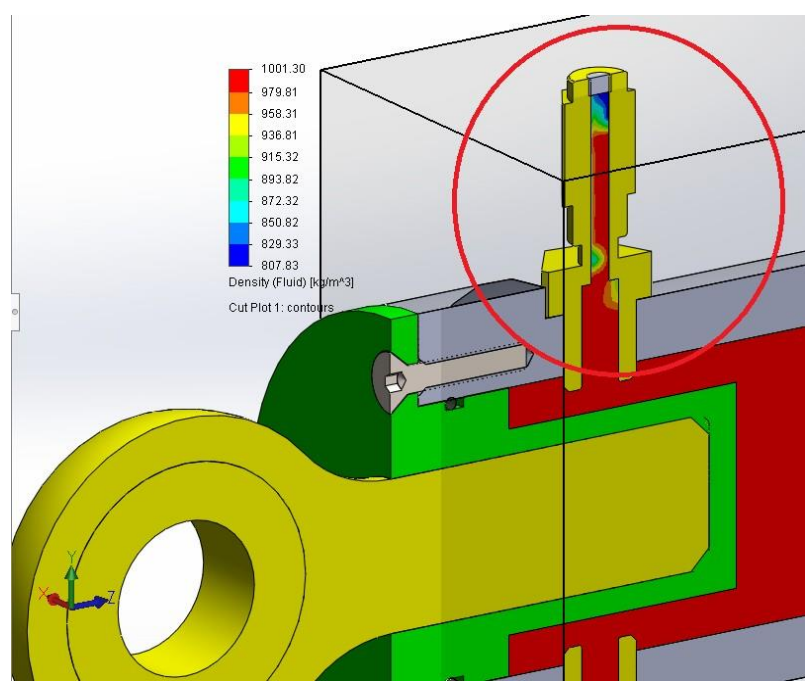

(a) velocity. The high peaks, shown by encircled area marked C, could be caused by the harsh impact during operation. This meant that the impact force $(F)$ which the vehicle hits the pothole is directly proportional to the velocity (V) at which the piston travels

i.e. $(\mathrm{F} \alpha \mathrm{V})$, this influenced the fluid's velocity during operation. Since the drop in pressure of the system is proportional to the square of velocity, the drop in pressure due to the increase in average speed was interpreted as the transfer of Kinetic Energy from the random molecular motion to the mean motion. As the fluid flowed through the constricted passage of the reserve cylinder, it accelerates resulting in an increased Kinetic Energy. This affected the fluid density during damping shown in Fig. 9.

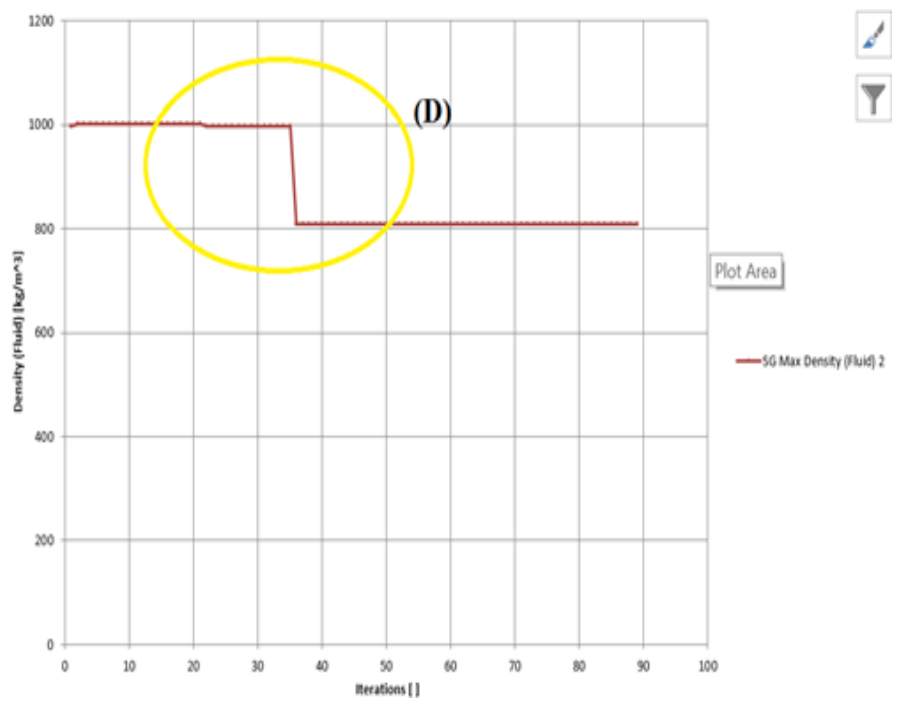

(b)

Figure 9 (a) Density simulation schematic with contours (b) Density against iteration

From compressible flow and assuming the process is isothermal and a thermodynamic process, the density of the fluid is directly proportional to the pressure $(\mathrm{P} \alpha \rho)$. As the system dampens vibratory oscillations during impact the system experiences a drop in pressure between iterations 0 40(t) as shown in Fig. 9 (b). This drop results a decrease in the fluid's density, hereafter the density stabilizes. The density of the fluid is less dense during iterations 40-90(t) compared to 
iterations $0-30(\mathrm{t})$, this trend follows the same trend of the system's pressure.-When the system experiences high pressure, $P_{1}$ the density of the fluid is more dense, $\rho_{1}$ and when the pressure is low, $P_{2}$ the density is less dense, $\rho_{2}$. The behaviour of the density is dependent on the system pressure as adherence to the principle of compressible flow as shown in Fig.9 (a).
Hence with the fluctuation in density the Kinetic Energy also fluctuates. This region of density change is shown by the encircled area marked D in the results plot as shown in Fig.9 (b). This affected the energy of the turbulent fluid during damping as shown in Fig. 10.

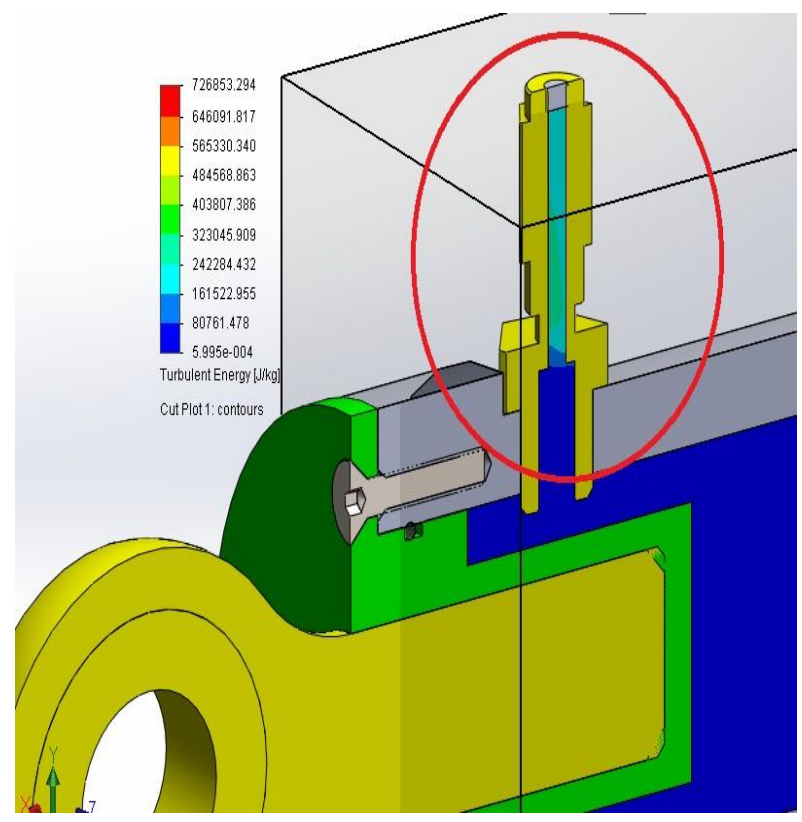

(a)

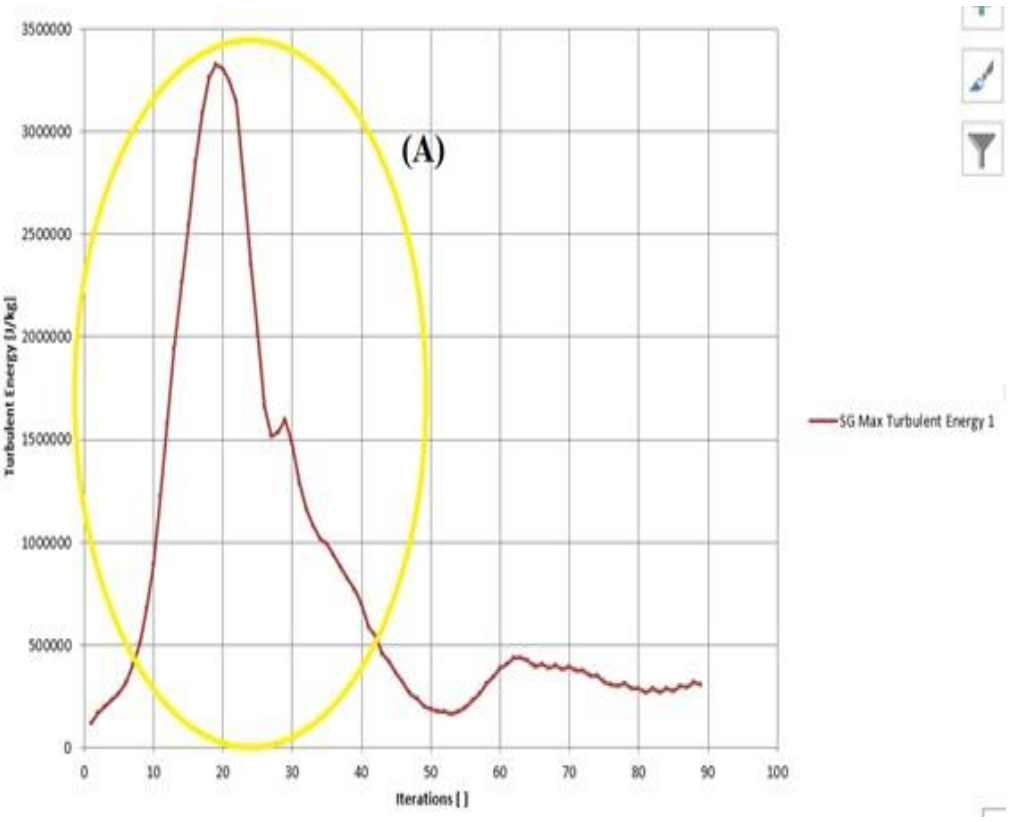

(b)

Figure 10 (a) Turbulent energy simulation schematic with contours (b) Turbulent Energy during damping process.

Fluid turbulence is the violent and unsteady movement of a fluid as shown in Fig.10. By interpretation of the damping system, during operation, results in high turbulent Energy during iteration $0-40(\mathrm{t})$ of which hereafter it slightly fluctuates until it stabilizes as shown in Fig. 10 (b). Now the iteration 040(t) represents the system's action area and by theoretical study it is during this initial motion of the fluid as it enters and exits the reserve cylinder that the fluid experiences high resistance as shown in Fig. 10 (b). As the system operates and while the fluid overcomes the resistance turbulent flow is experienced. The turbulent flow result in eddying motion of molecules of the fluid henceforth a large part of the Mechanical Energy goes to the formation of these eddies which eventually results in energy dissipated as shown in Fig. 10 (a). The turbulent Energy produced shown by the encircled area marked A is characterized by the unsteady eddying motion of the molecules as shown in Fig. 10 (b). These eddies are in constant motion with respect to each other, at any instant the magnitude of the eddies resulted in fluctuations in the pressure and velocity flow of the fluid and according to the streamline trend of the velocity result it was observed that at high velocities the eddies interact with each other as they move thus resulting in exchange and transfer of energy as shown in Fig. 10 (b). The turbulent Energy is at peak during iteration 0-40(t) because it is during this action phase that the system experiences high turbulence and thus having high Energy dissipation which can be conserved as shown in Fig. 10 (b).

\section{CONCLUSION AND RECOMMENDATION}

The current study was aimed at modelling and simulating the ignored parameters during automotive shock absorber damping process. The current models and simulation in automotive energy harvesting focus on limited parameters without considering other major energy parameter such as kinetic energy, potential energy, density of fluid, pressure during damping and change in temperature which affect the fluid viscosity. This study focused on modelling and simulating this parameters during damping. It was shown that the Kinetic Energy is dependent and directly proportional to the system's mass and velocity during damping. It was also shown that even though the vehicle's mass is constant, the vehicle's Kinetic Energy is at its highest when the vehicle's dampers dampens vibratory oscillations at high velocities while transferring the energy of the spring motion to the damper whist ensuring ride comfort, as illustrated by the excitation and response portion encircled. It was also observed from the pressure simulation obtained results that the pressure difference between iterations $0-5(\mathrm{t})$ showed a large drop and from iterations 5-40(t) the pressure experienced fluctuations of maximum and minimal peaks until it stabilized. It was also observed that the drop in pressure of the system is proportional to the square of velocity and the drop in pressure due to the increase in average speed was interpreted as the transfer of Kinetic Energy during 
damping. It was also revealed that the turbulent flow of fluid result in eddying motion of molecules of the fluid henceforth a large part of the Mechanical Energy goes to the formation of these eddies which eventually results in energy dissipated as observed. The turbulent Energy produced shown by the encircled area marked A is characterized by the unsteady eddying motion of the molecules during damping. Though the current study focused on ignored model in previous modelling and simulation of a twin damping during operation, the compressibility potential of the fluid can be considered in the process of developing future related models related to a damper systems i.e. damper performance characteristic models. Furthermore for analysis purposes the function $\left(\left(\log _{e}\right)\right.$ can be studied in detail to understand the correlation it has with the system's Energy potential. For contribution towards the sustainability of Energy, analysis can be made to investigate which mechanical systems can be powered by the conserved Energy under different damper operations which can help in selecting suitable Regenerative technology that can harvest the quantity of Energy obtained from damper systems.

\section{ACKNOWLEDGEMENT}

This material is based on the work which is supported financially by the Vaal University of Technology (VUT).

\section{REFERENCES}

[1] N.B KATE, T.A JADHAV, 2013. Mathematical Modelling of an Automotive Damper. International Journal of Engineering Research. Volume 2, Issue 7.

[2] HASNOLIZAR ZAKARIA ET AL, 2017. Energy Harvesting Mathematical Model on Vehicle Shock Absorber. International journal of Scientific Research Engineering and Technology. Volume 6. Issue 11.

[3] KIRK SHAWN RHOADES 2006. Development and Experimental Verification of a Parametric Model for an Automotive Damper. August 2006.

[4] SREEDHU S NAIRE, 2013. Empirical Modelling of Automotive Damper Curves and Development of Shape Factors. September 2013.

[5] BARBARA L ET AL, 2016. From Analysis to Energy Harvesting System Design Application to Car Suspension. International Engineering Journal of Manufacturing. Volume 10. Issue 50.

[6] PETER MÚČKA. 2016. Energy-harvesting potential of automobile suspension, Vehicle System Dynamics, 54:12, 1651-1670, DOI: 10.1080/00423114.2016.1227077.

[7] ZHANG JIN-QIN, PENG ZHI-ZHOA, ZHANG LEI, ZHANG YU. 2013. A Review-Regenerative

[8] Suspension Systems for Vehicles. Proceedings of the World Congress on Engineering 2013 Volume III, ISBN: 978-988-19252-9-9

[9] VELINSKY, STEVEN A. AND WHITE, ROBERT A. 1980. Vehicle Energy Dissipation Due to Road
Roughness", Vehicle System Dynamics, 1980, 9:6, pp. 359-384.

[10] LI, Z.; BRINDAK, Z.; ZUO, L. 2011. Modelling of an Electromagnetic Vibration Energy Harvester with Motion Magnification. In Proceedings of the ASME 2011 International Mechanical Engineering Congress and Exposition, Denver, CO, USA, 11-17 November 2011; pp. 285-29.

[11] NAKANO, K.; SUDA, Y.; NAKADAI, S. 2015. Selfpowered active vibration control using continuous control input. JSME International Journal. Ser. C 2000, 43, 726-731.

[12] SABZEHGAR, R.; MARAVANDI, A.; MOALLEM, M. 2014. Energy Regenerative Suspension Using an Algebraic Screw Linkage Mechanism. IEEE/ASME Trans. Mechatronic. 2014, 19, 1251-1259

[13] ZHANG JIN-QIU ET AL. 2013. A review on energyregenerative suspension systems for vehicles". In: Proceedings of the World Congress on Engineering. Vol. 3. 2013.

[14] XD XIE AND Q WANG. 2015. Energy harvesting from a vehicle suspension system”. In: Energy 86 (2015)

[15] ZHIGANG FANG ET AL. 2013. Experimental study of damping and energy regeneration characteristics of a hydraulic electromagnetic shock absorber. In: Advances in Mechanical Engineering 5 (2013). 